\title{
Appraisal of a Model to Estimate Survival in Ambulatory Patients with Hepatocellular Carcinoma Classified as Barcelona Clinic Liver Cancer Stage B
}

\author{
Jeong-Ju Yoo ${ }^{1}$, Su Jong Yü ${ }^{2}$, Bora Lee ${ }^{3}$, Eun Ju $\mathrm{Cho}^{2}$, Jeong-Hoon Lee ${ }^{2}$, Sang Gyune $\mathrm{Kim}^{1}$, Yoon Jun $\mathrm{Kim}^{2}$, Young Seok \\ $\mathrm{Kim}^{1}$, and Jung-Hwan Yoon ${ }^{2}$
}

${ }^{1}$ Department of Internal Medicine, Soonchunhyang University School of Medicine, Bucheon, ${ }^{2}$ Department of Internal Medicine and Liver Research Institute, Seoul National University College of Medicine, and ${ }^{3}$ Department of Statistics, Graduate School, Chung-Ang University, Seoul, Korea

Background/Aims: Patients with an intermediate stage of hepatocellular carcinoma (HCC) represent a highly heterogeneous population; therefore, many models have been proposed to predict the survival of these patients. The aim of this study was to evaluate the prognostic performance of a novel subclassification for tumors classified as BarceIona Clinic Liver Cancer (BCLC) stage B using the Model to Estimate Survival in Ambulatory HCC patients (MESIAH). Methods: This analysis was based on 377 patients with HCC treated at Seoul National University Hospital (training cohort) and 189 patients at the Soonchunhyang University Bucheon Hospital (validation cohort). Four subclassification systems were tested: MESIAH; original BCLC B subclassification (B1, B2, B3, and B4); modified model A (B1, B2, and B3+B4); and modified model B (B1, B2+B3, and B4). Results: Median survival progressively decreased from stage B1 through stages B2 to B3 according to the new MESIAH subclassification ( $p<0.001$ ). Moreover, significantly different survival among contiguous stages was observed. In the multivariable Cox regression, the MESIAH subclassification was an independent predictor of overall survival $(p<0.001)$. In terms of discrimination and calibration, MESIAH performed better than the original BCLC B subclassification, modified model A and modified model B. Conclusions: The MESIAH model would be an effective tool for stratifying heterogeneous BCLC stage B cancer, and the ability of this model to predict survival is better than that of the other previously proposed models. (Gut Liver 2020;14:377-386)

Key Words: Carcinoma, hepatocellular; Survival; Classification

\section{INTRODUCTION}

The stage classification of malignant tumors, especially hepatocellular carcinoma (HCC), has very important meaning. ${ }^{1,2}$ Basically, it is important because it predicts the duration of life. Furthermore, it can help determine treatment options by comparing the results of each treatment method. The most commonly used classification method for HCC is the Barcelona Clinic Liver Cancer (BCLC) classification. ${ }^{3}$ The BCLC classification is divided into early, intermediate and advanced HCC, and the proportion of intermediate stage, stage $\mathrm{B}$, is especially high, at approximately 50\%. ${ }^{4}$ Transarterial chemoembolization (TACE) is the standard treatment option in stage B; however, the responses to treatment and prognosis are very different for each patient. ${ }^{5,6}$ Because of this heterogeneity of stage B, Bolondi et al. ${ }^{4}$ first proposed to refine the intermediate stage in 2012. Intermediate stage of HCC was subclassified from B1 to B4 using up to seven rules and Child-Pugh score. ${ }^{4}$ After this study, many modified subclassification systems based on Bolondi's original subclassification have been published. ${ }^{7-9}$

The Model to Estimate Survival in Ambulatory HCC Patient (MESIAH) is a prognosis prediction system for HCC, first published in 2012. ${ }^{10}$ The classification was created at the Mayo Clinic using baseline demographic, clinical and tumor characteristics in patients with HCC. Unlike other classification systems, it is composed of only objective parameters and has the advantage of reflecting liver function, tumor burden and tumor biology. Indeed, MESIAH was superior to other prognostic prediction systems when validated for 904 HCC patients. ${ }^{11}$ Nevertheless, to the best of our knowledge, MESIAH has not been compared to

Correspondence to: Su Jong Yu

Department of Internal Medicine and Liver Research Institute, Seoul National University College of Medicine, 101 Daehak-ro, Jongno-gu, Seoul 03080, Korea Tel: +82-2-2072-2228, Fax: +82-2-743-6701, E-mail: ydoctor2@hanmail.net

Received on April 5, 2019. Revised on May 11, 2019. Accepted on May 23, 2019. Published online September $20,2019$. pISSN 1976-2283 eISSN 2005-1212 https://doi.org/10.5009/gnl19112

(c) This is an Open Access article distributed under the terms of the Creative Commons Attribution Non-Commercial License (http://creativecommons.org/licenses/by-nc/4.0) which permits unrestricted non-commercial use, distribution, and reproduction in any medium, provided the original work is properly cited. 
other subclassification systems in stage B patients. ${ }^{12}$

The aim of this study was to evaluate the prognostic performance of a novel subclassification for BCLC B intermediate stage using MESIAH and to compare it with Bolondi's original subclassification and other modified classifications.

\section{MATERIALS AND METHODS}

\section{Patients}

A consecutive series of 1,385 HCC patients who were diagnosed with HCC for the first time were investigated at two tertiary hospitals (Seoul National University Hospital [SNUH], Seoul, Korea and Soonchunhyang University Bucheon Hospital [SCH], Bucheon; Korea) between January 2004 and December 2010. We retrospectively enrolled 566 patients who had BCLC stage B disease and at the time of their initial diagnosis and undergone TACE as initial treatment. Patients were excluded if they had (1) previous or current malignancy other than HCC, (2) history of loco-regional or systemic therapy prior to initial diagnosis, or (3) observation period less than 4 weeks. Finally, 377 patients treated in SNUH were assigned to the training cohort and 189 patients treated in SCH were assigned to the validation cohort.

Written consent was waived, because the participants were to remain anonymous and the data were analyzed anonymously. The study protocol conformed to the ethical guidelines of the World Medical Association Declaration of Helsinki and the study protocol was approved by the Institutional Review Boards of both hospitals (IRB numbers: H-1409-025-607, SCHBC 201809-011-001).

Table 1. Baseline Characteristics

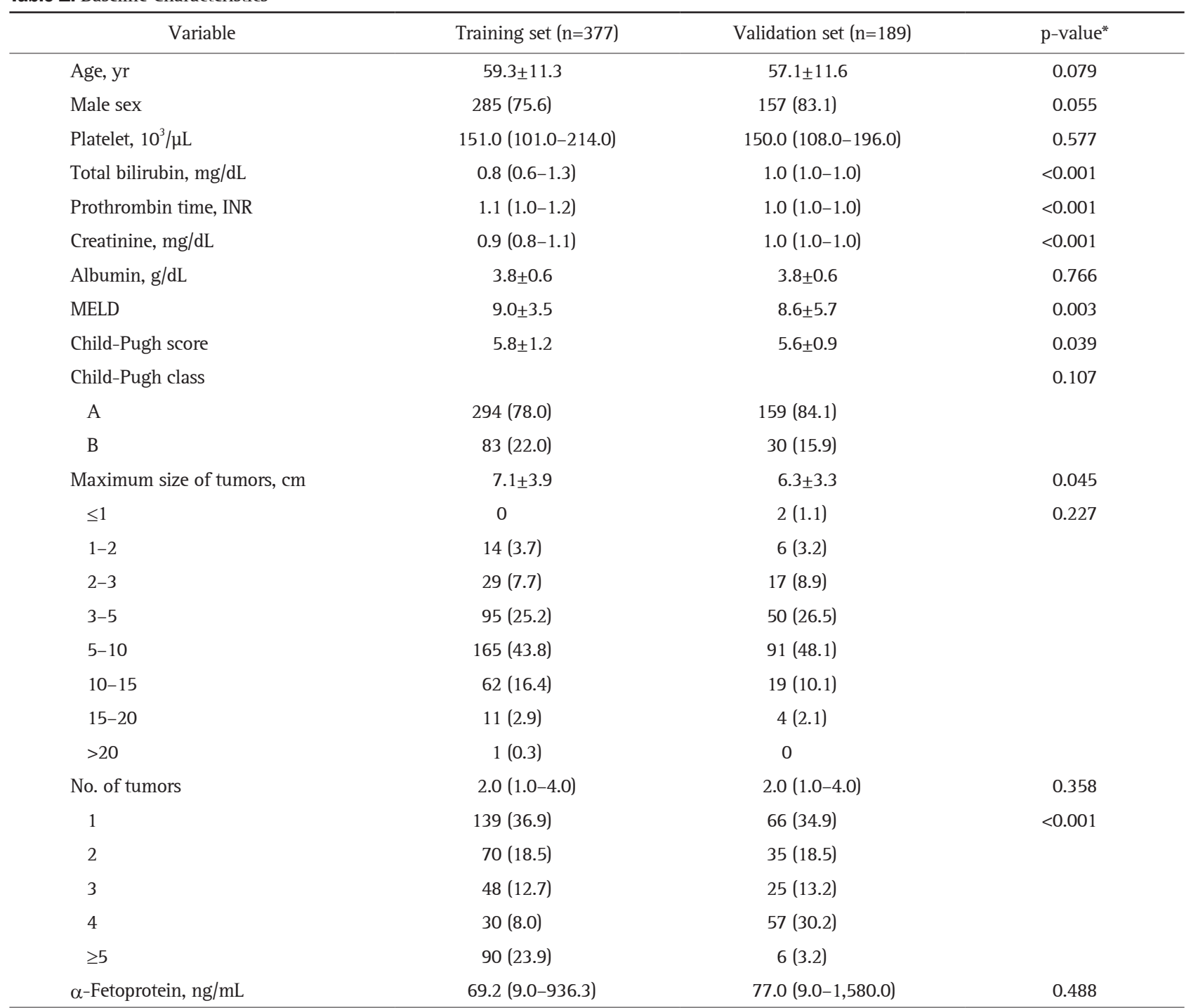

Data are presented as mean $\pm \mathrm{SD}$, number (\%), or median (interquartile range).

INR, international normalized ratio; MELD, Model for End-Stage Liver Disease.

*p-values were calculated by Student t-test or the Mann-Whitney U test for continuous variables and by the chi-square test for categorical variables. 


\section{Diagnosis of HCC, definition of intermediate stage, and procedure of TACE}

Diagnosis and staging of HCC were based on the American Association for the Study of Liver Diseases criteria and BCLC staging system. ${ }^{13,14}$ Intermediate stage (BCLC stage B) of HCC was defined as multinodular tumors without vascular invasion or extrahepatic metastasis, performance status $0 .{ }^{5}$ A single large tumor more than $5 \mathrm{~cm}$ beyond Milan criteria, which is not suitable for surgery or radiofrequency ablation, was also classified as BCLC stage B. ${ }^{9,15-17}$

Superselective TACE was performed when possible through the lobar, segmental or subsegmental arteries, depending on the tumor distribution and hepatic function reserve. ${ }^{18-20}$ The extent of chemoembolization was individually adjusted by using a superselective catheterization technique. The volume of iodized oil ranged from 2 to $12 \mathrm{~mL}$, and the amount of doxorubicin ranged from 10 to $60 \mathrm{mg}$. The total amount of cisplatin used ranged from 50 to $100 \mathrm{mg}$ depending on the patient's body weight and the rate of infusion. Following initial treatment, patients were monitored using dynamic computed tomography (CT) or magnetic resonance imaging every 1 to 3 months with repeated TACE performed on demand based on the findings of follow-up $\mathrm{CT}$, including local recurrence and liver function.

\section{Bolondi's subclassification and MESIAH score}

Original BCLC B subclassification was based on Bolondi's proposal. ${ }^{4}$ Modified models were divided into three stages as follows using the original BCLC B subclassification system: modified model A, B1 versus B2 versus B3+B4; and modified model $\mathrm{B}, \mathrm{B} 1$ versus $\mathrm{B} 2+\mathrm{B} 3$ versus $\mathrm{B} 4 .^{21}$ The MESIAH score was calculated according to previous studies: ${ }^{10}$

MESIAH score=0.232 $\times$ (age in decades)+0.099 $\times$ (Model for EndStage Liver Disease [MELD] score)-0.391×(albumin)+0.290×(tumor size) $+0.153 \times$ (tumor number) $+1.122 \times($ vascular invasion $)+1.130 \times$ (metastasis)+0.082×( $\alpha$-fetoprotein) +1 .

\section{Statistical analyses}

Clinical, laboratory and radiologic records of all patients were retrospectively reviewed. Frequencies and percentages were used for descriptive statistics. Statistical differences between the two groups were investigated using the chi-square test and Student t-test. Patient survival probability was estimated using the Kaplan-Meier method and differences between the curves were compared using the log-rank test. Overall survival (OS) was defined as the time from the date of HCC diagnosis until the date of death from any causes. We performed discrimination and calibration to see how each model predicts the OS of the patients. The main analysis tool used for survival was the Cox proportional hazards model. All statistical analyses were performed using R version 3.3.3 (the R Foundation for Statistical Computing, Vienna, Austria). Statistical significance was defined as a $\mathrm{p}<0.05$.

We used multivariable Cox proportional hazard analysis to see how the survival rate differed according to the stage of each model. Model performance was evaluated with respect to discrimination and calibration. For discrimination, c-statistics, linear trend chi-square, and incremental area under the curve were used. A staging system with c-statistics of 1 would have predicted the correct OS in all patients; c-statistics of 0 would mean that the incorrect outcomes were predicted in all patients; c-statistics of 0.5 would imply that the system correctly predicted the OS 50\% of the time. For calibration, likelihood ratio chisquare, and Somer's Dxy was used. The same methods, discrimination and calibration were performed for the validation set.

\section{RESULTS}

\section{Baseline characteristics}

This study analyzed a total of 566 patients with intermediate stage HCC who underwent TACE. Finally, 377 patients treated in SNUH were assigned to the training cohort and 189 patients

Table 2. Subclassification of Each Staging System

\begin{tabular}{ccc}
\hline \multicolumn{1}{c}{ Variable } & $\begin{array}{c}\text { Training set } \\
(\mathrm{n}=377)\end{array}$ & $\begin{array}{c}\text { Validation set } \\
(\mathrm{n}=189)\end{array}$ \\
\hline Bolondi's BCLC B subclassification & & \\
1 & $100(26.5)$ & $78(41.3)$ \\
2 & $201(53.3)$ & $93(49.2)$ \\
3 & $26(6.9)$ & $10(5.3)$ \\
4 & $50(13.3)$ & $8(4.2)$ \\
BCLC B subclassification (revised-A) & & \\
1 & $100(26.5)$ & $78(41.3)$ \\
2 & $201(53.3)$ & $103(54.5)$ \\
3 & $76(20.2)$ & $8(4.2)$ \\
BCLC B subclassification (revised-B) & & \\
1 & $100(26.5)$ & $78(41.3)$ \\
2 & $227(60.2)$ & $93(49.2)$ \\
3 & $50(13.3)$ & $18(9.5)$ \\
MESIAH staging & & \\
1 & $12(3.2)$ & $8(4.2)$ \\
2 & $113(30.0)$ & $66(34.9)$ \\
3 & $191(50.6)$ & $92(48.7)$ \\
4 & $58(15.4)$ & $20(10.6)$ \\
5 & $3(0.8)$ & $3(1.6)$ \\
2 & & \\
\hline & $125(33.2)$ & $74(39.1)$ \\
& $191(50.6)$ & $92(48.7)$ \\
& & $23(12.2)$ \\
\hline
\end{tabular}

Data are presented as number (\%).

BCLC, Barcelona Clinic Liver Cancer; MESIAH, Model to Estimate Survival in Ambulatory Hepatocellular Carcinoma Patients. 
treated in SCH were assigned to the validation cohort. The baseline characteristics of the patients enrolled in the study are reported in Table 1. The median age of the patients was $58.4 \pm 11.4$ years, and 442 patients (78.1\%) were males. The mean tumor size was $6.6 \pm 3.5 \mathrm{~cm}$, and the median number of tumors in each patient was 2. Four hundred fifty-three patients (80.0\%) were classified as Child-Pugh class A, and 113 patients (20.0\%) were Child-Pugh class B. The mean MELD score was $8.8 \pm 4.2$ points. The distributions of patient characteristics were similar between the training set and validation set, except for MELD score, Child-Pugh score and maximal size of tumors.

\section{Distributions of the patients according to each subclas- sification system}

The enrolled intermediate stage HCC patients were reclassified according to their respective subclassification staging systems (Table 2, Supplementary Fig. 1). Unlike other subclassification stages, MESIAH is a continuous variable, so we divided patients into five groups according to their MESIAH score. Based on the existing literature, validated in Korean patients with HCC,
MESIAH has been categorized as follows: group 1, score 2.187 to 3.521 ; group 2, score 3.526 to 4.200 ; group 3, score 4.201 to 5.039; group 4, score 5.041 to 6.063 ; and group 5, score 6.072 to 10.093. Of these, groups 1 and 2 and groups 4 and 5 had similar characteristics and prognosis; they were merged into one group. Therefore, the final revised MESIAH groups were as follows: revised group 1, 2.187 to 4.200; revised group 2, 4.201 to 5.039; and revised group 3, 5.041 to 10.093 . The characteristics of subclassification group 1, 2 and 3 of BCLC B HCC are shown in Supplementary Table 1.

\section{Survival according to each subclassification system}

The Kaplan-Meier curve analyses of OS in the training and validation sets are shown in Fig. 1A (training set) and Fig. 1B (validation set). Survival rate and number at risk of the patients are shown in Supplementary Tables 2 (training set) and 3 (validation set). In the training set, the median survival decreased from stage B1 through B2, B3, and B4 in Bolondi's original subclassification system. Stages B1 and B2 showed a significant difference $(p=0.001)$, but no significant difference was found
A BCLC B subclassification

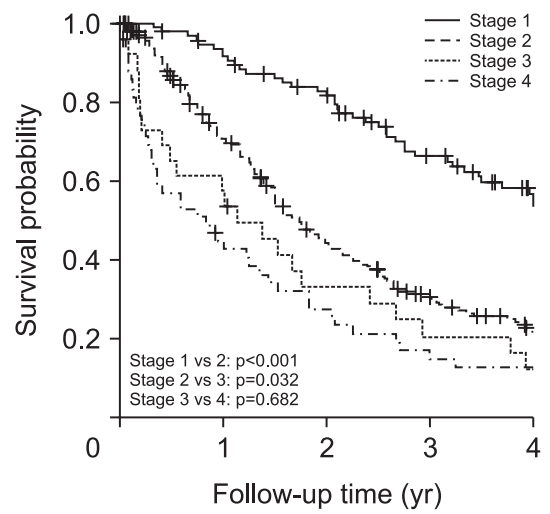

MESIAH staging

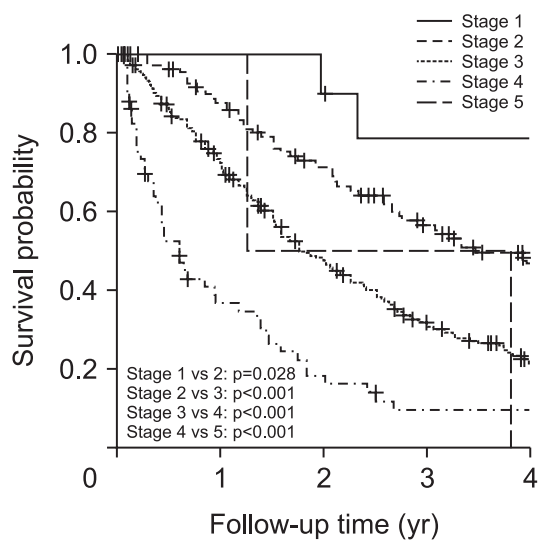

BCLC B subclassification (revised-A)

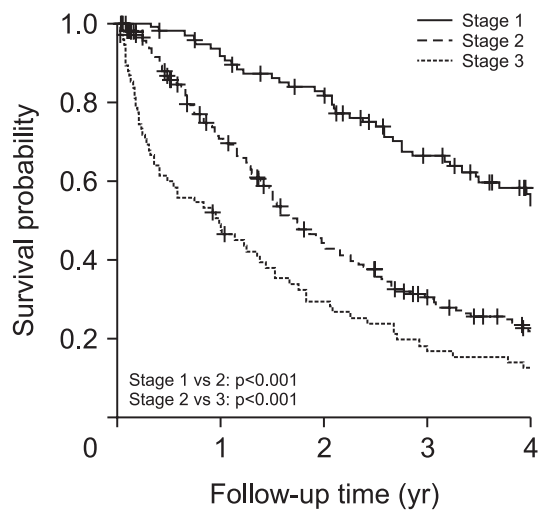

Revised MESIAH staging

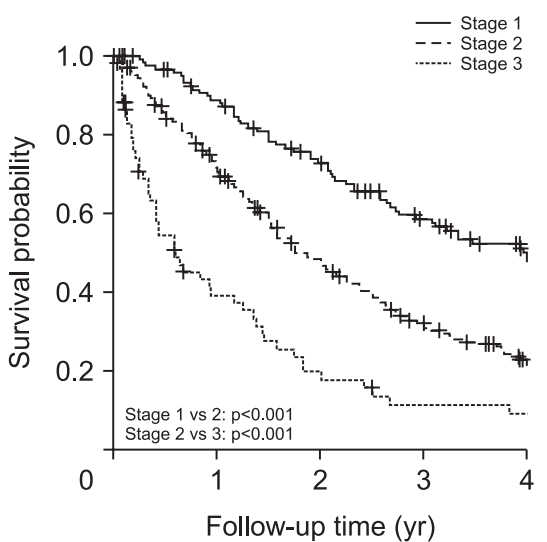

BCLC B subclassification (revised-B)

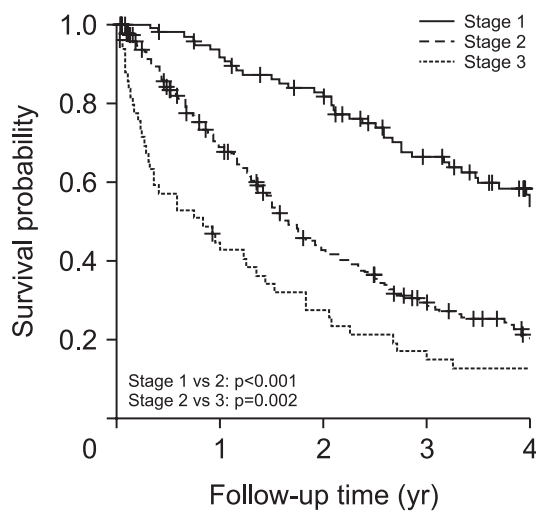

Fig. 1. Probability of overall survival according to each subclassification system in the validation set. Kaplan-Meier survival plots stratified by each system group according to each model in the (A) training set and (B) validation set.

BCLC, Barcelona Clinic Liver Cancer; MESIAH, Model to Estimate Survival in Ambulatory Hepatocellular Carcinoma Patients. 
B

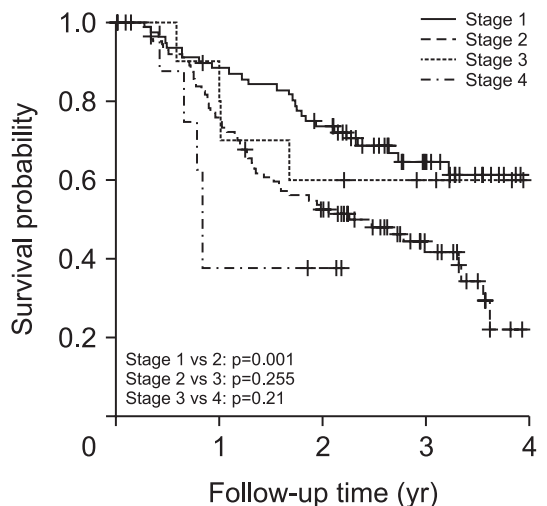

MESIAH staging

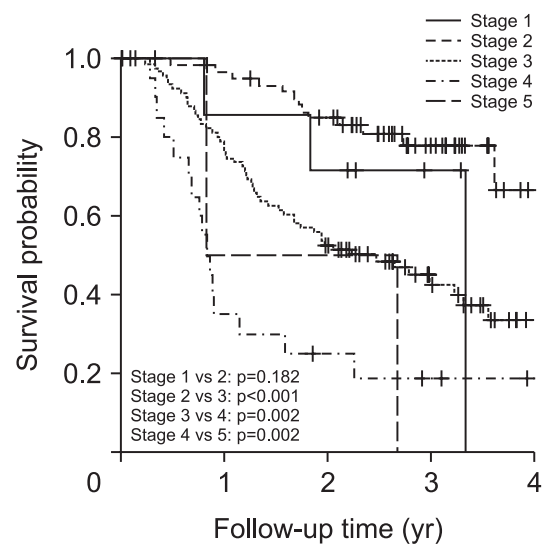

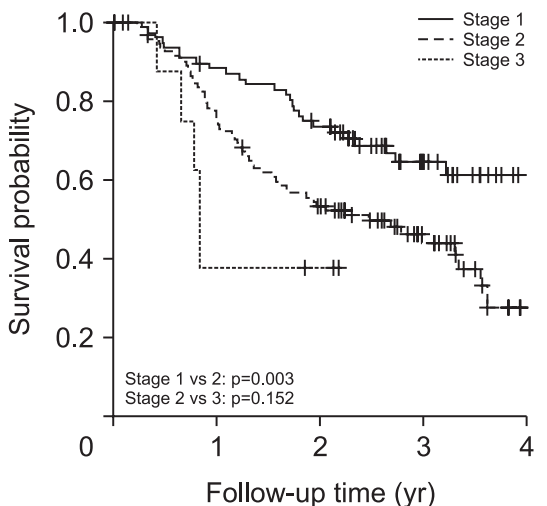

Revised MESIAH staging

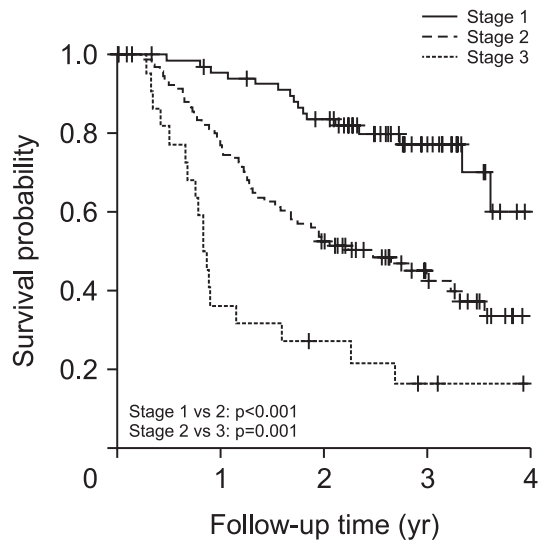

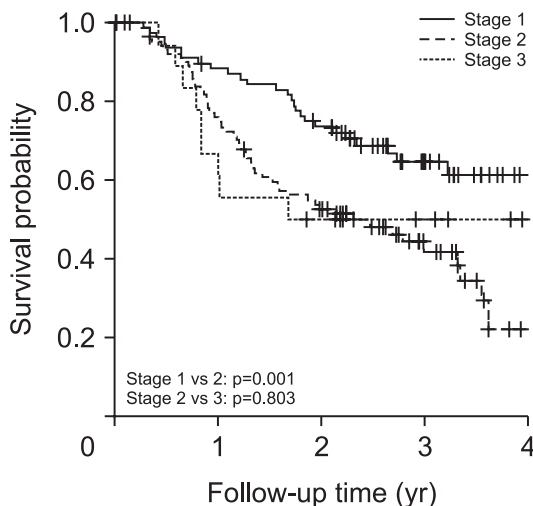

Follow-up time (yr)

Fig. 1. Continued.

in the other stages. In the case of the revised A model and the revised B model that modified Bolondi's model, there was a significant difference between stages B1 and B2, but no significant difference was found between stages 2 and 3. On the other hand, for the revised MESIAH model, median survival progressively decreased from stage 1 through stage 2 and stage 3. Moreover, we observed significantly different survival between contiguous stages (stage 1 vs stage $2, \mathrm{p}<0.001$; stage 2 vs stage 3 , $\mathrm{p}=0.001)$.

In the validation set, OS showed a similar pattern according to each subclassification system (Fig. 1B). In general, the OS tended to become shorter as the stage level increased. Only the revised MESIAH model showed significant differences between stages, while the other models did not show any significant difference between stages 2 and 3 .

\section{Predictors of survival}

The results of the univariate analyses in the training set are presented in Table 3. In the univariate analysis, all subclassification system including revised MESIAH model were associated with OS. Furthermore, high MELD score ( $\mathrm{p}<0.001)$, Child-Pugh class B $(\mathrm{p}<0.001)$, larger tumor size $(\mathrm{p}<0.001)$, increased number of tumors $(p=0.005)$ and higher $\alpha$-fetoprotein $(p<0.001)$ were associated with survival. However, in multivariable Cox regression analysis, only the revised MESIAH model and the revised B model were associated with OS, while other subclassification systems did not show significant survival differences for each stage (Table 4). In the validation set, the results of multivariable Cox regression analysis were similar, and only the revised MESIAH model was a significant factor associated with OS (Table 5).

\section{Discrimination and calibration of each subclassification system}

The overall c-statistic of the revised MESIAH model was 0.645 (95\% confidence interval [CI], 0.613 to 0.677 ) in the training set, and 0.683 (95\% CI, 0.631 to 0.677) in the validation set (Tables 4 , 5). When compared with other subclassification system, the overall c-statistic of the revised MESIAH model was superior to that of other models. Similarly, the likelihood ratio chi-square values were higher and the Akaike information criterion was lower for MESIAH than those for the other subclassification systems. 
Table 3. Cox Proportional Hazard Regression Analysis for Overall Survival in the Training Set

\begin{tabular}{|c|c|c|}
\hline \multirow{2}{*}{ Variable } & \multicolumn{2}{|c|}{ Univariate } \\
\hline & HR $(95 \%$ CI) & p-value \\
\hline Age, yr & $0.99(0.98-1.00)$ & 0.124 \\
\hline Male sex & $0.86(0.66-1.13)$ & 0.294 \\
\hline MELD & $1.08(1.05-1.10)$ & $<0.001$ \\
\hline Child-Pugh class B vs A & $2.17(1.66-2.84)$ & $<0.001$ \\
\hline Maximum size of tumors, $\mathrm{cm}$ & $1.11(1.08-1.15)$ & $<0.001$ \\
\hline No. of tumors & $1.07(1.02-1.12)$ & 0.005 \\
\hline $\begin{array}{l}\alpha \text {-Fetoprotein } \\
\quad(>200 \mathrm{ng} / \mathrm{mL} \text { vs }<200 \mathrm{ng} / \mathrm{mL})\end{array}$ & $1.72(1.34-2.21)$ & $<0.001$ \\
\hline \multicolumn{3}{|l|}{ BCLC B subclassification } \\
\hline 2 vs 1 & $2.54(1.81-3.55)$ & $<0.001$ \\
\hline 3 vs 1 & $4.03(2.45-6.63)$ & $<0.001$ \\
\hline 4 vs 1 & $4.57(3.02-6.93)$ & $<0.001$ \\
\hline \multicolumn{3}{|c|}{ BCLC B subclassification (revised-A) } \\
\hline 2 vs 1 & $2.53(1.81-3.55)$ & $<0.001$ \\
\hline 3 vs 1 & $4.37(2.99-6.38)$ & $<0.001$ \\
\hline \multicolumn{3}{|c|}{ BCLC B subclassification (revised-B) } \\
\hline 2 vs 1 & $2.68(1.92-3.73)$ & $<0.001$ \\
\hline 3 vs 1 & $4.57(3.01-6.92)$ & $<0.001$ \\
\hline \multicolumn{3}{|l|}{ MESIAH staging system } \\
\hline 2 vs 1 & $3.09(0.97-9.88)$ & 0.056 \\
\hline 3 vs 1 & $5.61(1.78-17.68)$ & 0.003 \\
\hline 4 vs 1 & $12.46(3.86-40.18)$ & $<0.001$ \\
\hline 5 vs 1 & $6.25(1.04-37.59)$ & 0.045 \\
\hline \multicolumn{3}{|l|}{ Revised MESIAH staging } \\
\hline 2 vs 1 & $1.98(1.47-2.65)$ & $<0.001$ \\
\hline 3 vs 1 & $4.22(2.91-6.12)$ & $<0.001$ \\
\hline
\end{tabular}

HR, hazard ratio; CI, confidence interval; MELD, Model for End-Stage Liver Disease; BCLC, Barcelona Clinic Liver Cancer; MESIAH, Model to Estimate Survival in Ambulatory Hepatocellular Carcinoma $\mathrm{Pa}$ tients.

\section{DISCUSSION}

TACE is the standard therapy for patients with intermediate stage HCC. ${ }^{3}$ However, due to the heterogeneous patient population of BCLC B patients, tailored subclassification of intermediate stage is required to provide effective treatment for each patient. ${ }^{22}$ In the present study, we formulated a subclassification system using MESIAH, and validated its significance with an external group that underwent TACE as their initial treatment.

Bolondi's subclassification was originally created primarily with the aim of optimizing treatment allocation. ${ }^{4}$ An important focus of new subclassification system is the ability to distinguish patients who will benefit from TACE compared to other treatment options. Nevertheless, there are limitations to this Bolondi's classification. First, prognostic classification was only the secondary purpose in Bolondi's study, and predictive power was reported lower according to the populations. Second, subjective items were included in Bolondi's classification, for example, performance status, resulting in low reproducibility. Finally, there were some areas that did not match real practice among the treatment recommendations in Bolondi's classification. In the original Bolondi's classification, only best supportive care was recommended in stage B4. However, stage B4 generally has better prognosis than B3, and as TACE technology evolves, TACE can be effectively applied to $\mathrm{B} 4 .^{23-26}$ In addition, sorafenib is the primary treatment in Quasi-C, but TACE is often performed in this subgroup, varying in regions and countries especially in Asia. ${ }^{23}$

Recently, some authors tested the usefulness of the subclassification proposed by Bolondi et al. ${ }^{4}$ and proposed modified subclassification systems. ${ }^{7,9,27,28}$ Bolondi's classification showed very high predictive power in patients with untreated HCC. ${ }^{27}$ In contrast to untreated HCC, in a validation study of patients receiving TACE, only B1 showed significantly better survival than the other substages. ${ }^{21,28-30}$ In other stages, the prognostic power of Bolondi's systems was lost. ${ }^{7}$ Similar to previous studies, only B1 had significantly better prognosis, while B2, B3, and B4 showed no significant difference in our study. Nevertheless, it is very rare that patients with intermediate stage would not receive any treatment, and ethical problems can occur if the patient is not treated. Therefore, it is clinically meaningful to develop a prognostic system for patients receiving standard therapy TACE.

Ha et $a{ }^{21}$ attempted to increase the predictive power by grouping B3 and B4 in the Bolondi's classification. In our cohort, we suggested a revised model A and B because the difference between B2 and B3 as well as B3 and B4 were not significant. Subsequently, revised models A and B showed better predictive power than Bolondi's classification. Both Bolondi's model and the revised model included subjective items; therefore, we tried to apply a more reproducible and objective classification.

The MESIAH score consisted of only objective variables; therefore, it is highly reproducible. ${ }^{10,12,31}$ MESIAH scores which use MELD scores have been shown to be very effective in predicting prognosis of HCC, and it has been shown that stratification can be performed even within the same BCLC categories. $^{31-34}$ Therefore, we applied MESIAH as a new subclassification of BCLC B patients. Since MESIAH is originally a continuous variable, we classified MESIAH into five groups based on existing studies. ${ }^{12}$ Among five groups of MESIAH, we found that groups 1 and 2 and groups 4 and 5 had similar characteristics and prognosis. According to these results, we reclassified patients with BCLC B HCC into group 1 (MESIAH, 2.187 to 4.200 ), group 2 (4.201 to 5.039), and group 3 (5.041 to 10.093) using MESIAH scores. OS was hierarchized by our subclassification of BCLC B HCC patients. Our new subclassification system using MESIAH scores demonstrated good discrimina- 
Yoo JJ, et al: BCLC B Subclassification Using MESIAH Score 383

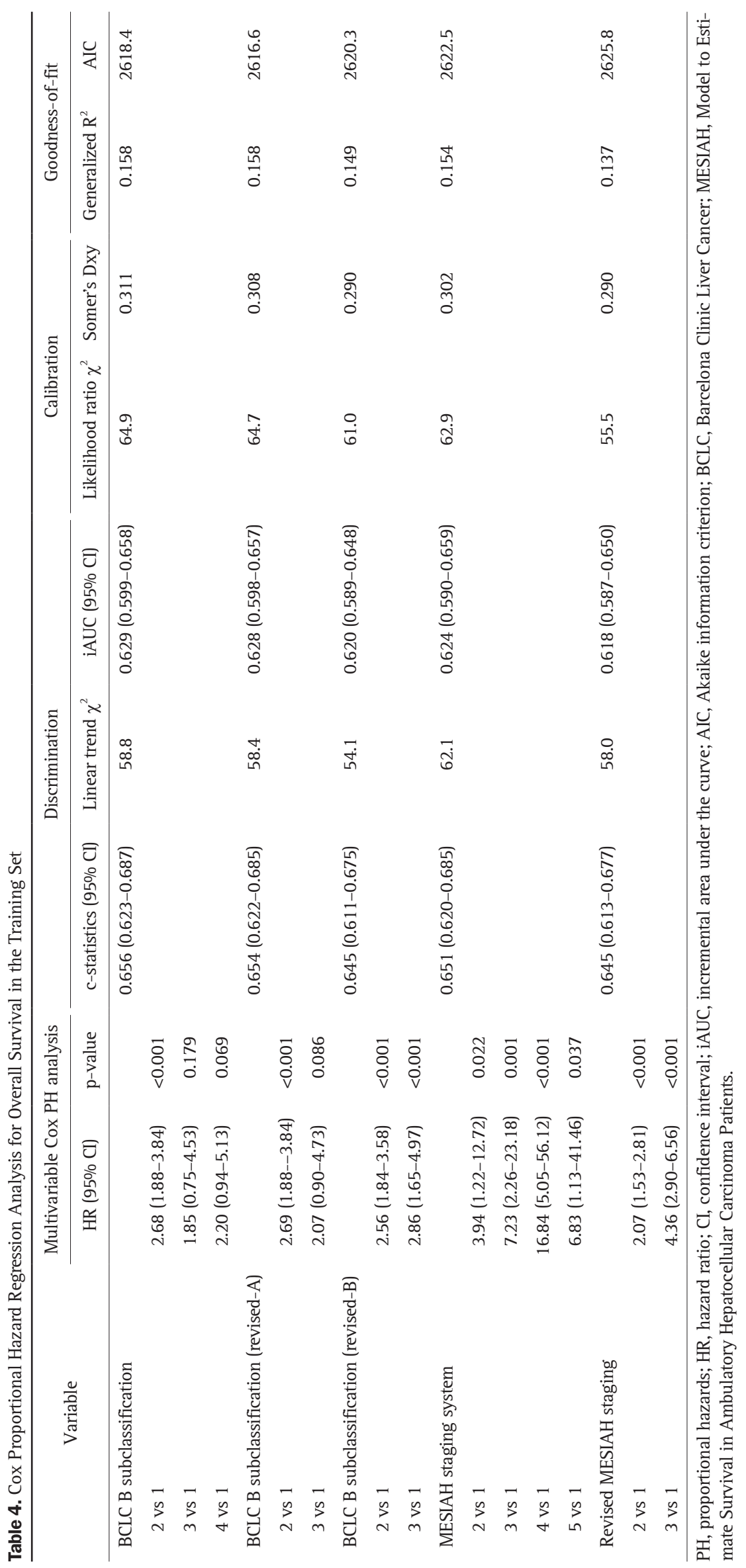




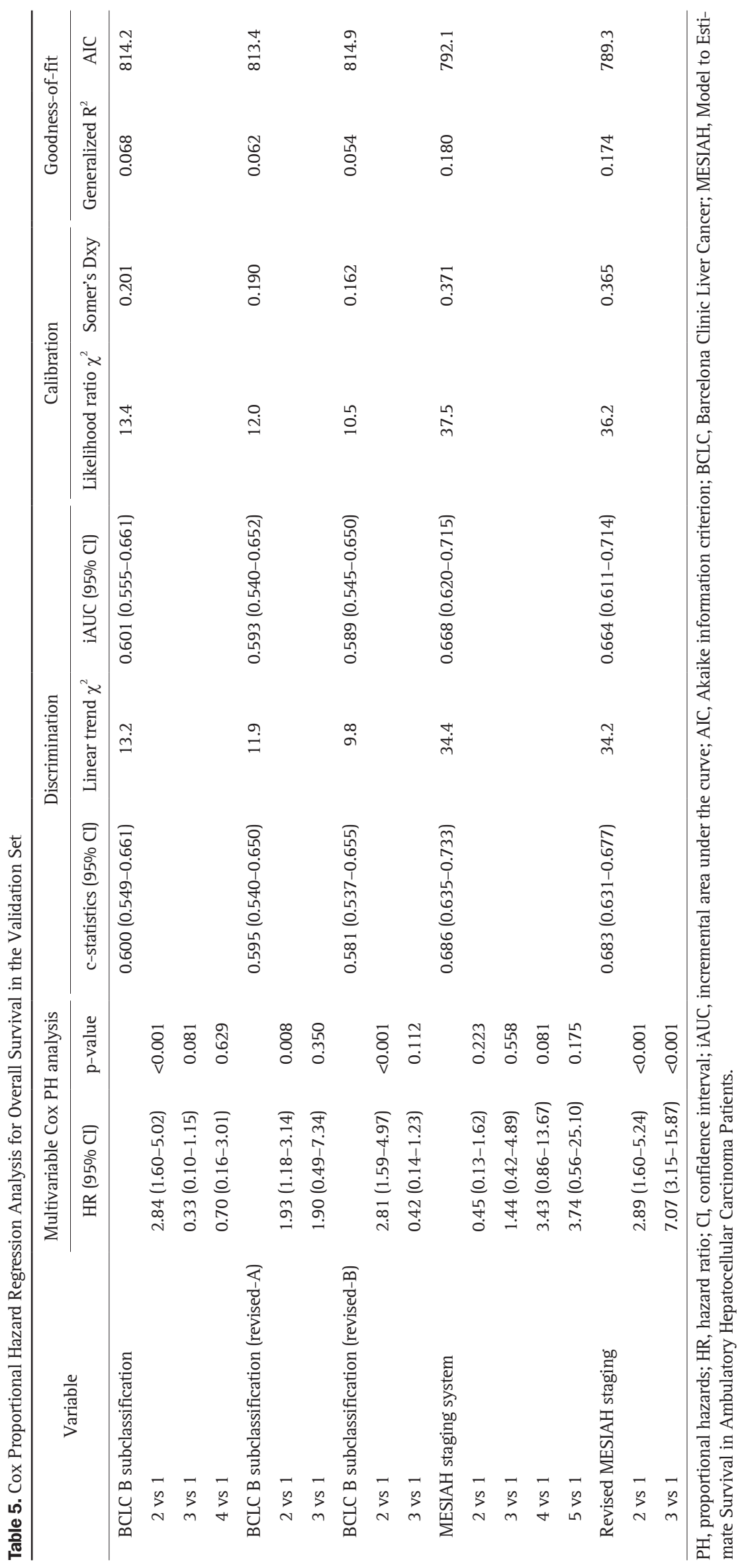


tion and calibration in predicting OS both in the training and validation cohorts. MEISAH score was originally developed in Western countries, but it proved efficacy and validated well in Asian patient cohort. ${ }^{10}$ Based on our findings, we believe that MESIAH score can be applied in a wide range of patients in real clinical practice regardless of race and country. The reason for the superiority of the MESIAH score to other systems is that it uses both the tumor factor and the MELD score, reflecting liver function. ${ }^{7}$ MELD score was reported to provide a finer tuning of prognosis in the BCLC B subclassification, and in particular in stages B1 and B2. ${ }^{10}$ The importance of the MELD score has been mentioned in other Western studies, and this study showed the need for a more refined assessment of liver function impairment through inclusion of MELD score. ${ }^{7,27,28}$

Limitations of our study include its retrospective nature, the homogenous treatment modality and incomplete knowledge of the clinical history of patients until death. Nevertheless, as noted earlier, considering that the proportion of untreated HCC is very small and TACE is the standard therapy in intermediate stage, our study data are likely to be closer to real practice. Previous studies have also been performed only in one homogenous institution. To overcome this limitation, we conducted a separate analysis on an external validation cohort. Third, although data are from large-volume liver centers in Asia, it remains possible that our results may not be pertinent for Western patients with HCC owing to differences in demographic findings including underlying causes of liver disease. Finally, the application of MESIAH score in clinical practice could not be evaluated in this study. The aim of subclassification in the BCLC stage B is not only to explain the prognosis of the patient, but also to match the better treatment option in the poor prognostic group instead of TACE. In this respect, MESIAH has the advantage of predicting prognosis, but there is a lack of analysis as to which treatment should be appropriate for each sub-stage.

In conclusion, our new BCLC B substaging system, using MESIAH as the main parameters, has excellent discriminatory power to subclassify TACE-treated patients into three prognostic substages. Although this study showed the good prognostic capability of the revised MELD scores, other future studies with data from longer periods and larger sample sizes are warranted to validate our results.

\section{CONFLICTS OF INTEREST}

No potential conflict of interest relevant to this article was reported.

\section{ACKNOWLEDGEMENTS}

The Biospecimens and data used in this study were provided by the Biobank of Seoul National University Hospital, a member of Korea Biobank Network.
The authors thank Eun-Ae Jung (Librarian, Medical Library, Soonchunhyang University Bucheon Hospital) for carefully proofreading the manuscript.

\section{AUTHOR CONTRIBUTIONS}

Conceptualization, writing - original draft: J.J.Y., S.J.Y. Methodology: E.J.C., J.H.L., S.G.K. Validation of data: Y.S.K. Formal analysis: B.L. Investigation: Y.J.K., J.H.Y. Visualization: B.L. Resources: E.J.C., J.H.L. Supervision: S.G.K. Approval of final manuscript: all authors.

\section{ORCID}

Jeong-Ju Yoo

https://orcid.org/0000-0002-7802-0381

Su Jong $\mathrm{Yu}$

https://orcid.org/0000-0001-8888-7977

Bora Lee

https://orcid.org/0000-0002-6322-5712

Eun Ju Cho

https://orcid.org/0000-0002-2677-3189

Jeong-Hoon Lee

https://orcid.org/0000-0002-0315-2080

Sang Gyune Kim

https://orcid.org/0000-0001-8694-777X

Yoon Jun Kim

https://orcid.org/0000-0001-9141-7773

Young Seok Kim

https://orcid.org/0000-0002-7113-3623

Jung-Hwan Yoon

\section{REFERENCES}

1. Pons F, Varela M, Llovet JM. Staging systems in hepatocellular carcinoma. HPB (Oxford) 2005;7:35-41.

2. Yu SJ. A concise review of updated guidelines regarding the management of hepatocellular carcinoma around the world: 20102016. Clin Mol Hepatol 2016;22:7-17.

3. Bruix J, Sherman M; American Association for the Study of Liver Diseases. Management of hepatocellular carcinoma: an update. Hepatology 2011;53:1020-1022.

4. Bolondi L, Burroughs A, Dufour JF, et al. Heterogeneity of patients with intermediate (BCLC B) hepatocellular carcinoma: proposal for a subclassification to facilitate treatment decisions. Semin Liver Dis 2012;32:348-359.

5. Forner A, Llovet JM, Bruix J. Hepatocellular carcinoma. Lancet 2012;379:1245-1255.

6. D’Avola D, Iñarrairaegui M, Pardo F, et al. Prognosis of hepatocellular carcinoma in relation to treatment across BCLC stages. Ann Surg Oncol 2011;18:1964-1971.

7. Biolato M, Gallusi G, Iavarone M, et al. Prognostic ability of BCLC-B subclassification in patients with hepatocellular cvarcinoma undergoing transarterial chemoembolization. Ann Hepatol 2018;17:110-118.

8. Zhang N, Gu J, Yin L, et al. Incorporation of alpha-fetoprotein (AFP) into subclassification of BCLC C stage hepatocellular carcinoma according to a 5-year survival analysis based on the SEER database. Oncotarget 2016;7:81389-81401.

9. Kudo M, Arizumi T, Ueshima K, Sakurai T, Kitano M, Nishida N. 
Subclassification of BCLC B stage hepatocellular carcinoma and treatment strategies: proposal of modified Bolondi's subclassification (Kinki Criteria). Dig Dis 2015;33:751-758.

10. Yang JD, Kim WR, Park KW, et al. Model to estimate survival in ambulatory patients with hepatocellular carcinoma. Hepatology 2012;56:614-621.

11. Park KW, Park JW, Choi JI, et al. Survival analysis of 904 patients with hepatocellular carcinoma in a hepatitis B virus-endemic area. J Gastroenterol Hepatol 2008;23:467-473.

12. Kim BH, Park JW, Nam BH, Kwak HW, Kim WR. Validation of a model to estimate survival in ambulatory patients with hepatocellular carcinoma: a single-centre cohort study. Liver Int 2014;34:e317-e323.

13. Edge SB, Compton CC. The American Joint Committee on Cancer: the 7th edition of the AJCC cancer staging manual and the future of TNM. Ann Surg Oncol 2010;17:1471-1474.

14. Heimbach JK, Kulik LM, Finn RS, et al. AASLD guidelines for the treatment of hepatocellular carcinoma. Hepatology 2018;67:358380.

15. Jung YK, Jung CH, Seo YS, et al. BCLC stage B is a better designation for single large hepatocellular carcinoma than BCLC stage A. J Gastroenterol Hepatol 2016;31:467-474.

16. Bruix J, Reig M, Sherman M. Evidence-based diagnosis, staging, and treatment of patients with hepatocellular carcinoma. Gastroenterology 2016;150:835-853.

17. Cho Y, Sinn DH, Yu SJ, et al. Survival analysis of single large ( $>5$ $\mathrm{cm}$ ) hepatocellular carcinoma patients: BCLC A versus B. PLoS One 2016;11:e0165722.

18. Kim HC, Lee JH, Chung JW, et al. Transarterial chemoembolization with additional cisplatin infusion for hepatocellular carcinoma invading the hepatic vein. J Vasc Interv Radiol 2013;24:274-283.

19. Choi JW, Kim HC, Lee JH, et al. Transarterial chemoembolization of hepatocellular carcinoma with segmental portal vein tumour thrombus. Eur Radiol 2017;27:1448-1458.

20. Yoo JJ, Lee JH, Lee SH, et al. Comparison of the effects of transarterial chemoembolization for advanced hepatocellular carcinoma between patients with and without extrahepatic metastases. PLoS One 2014;9:e113926.

21. Ha Y, Shim JH, Kim SO, Kim KM, Lim YS, Lee HC. Clinical appraisal of the recently proposed Barcelona Clinic Liver Cancer stage B subclassification by survival analysis. J Gastroenterol Hepatol 2014;29:787-793.
22. Kim JY, Sinn DH, Gwak GY, et al. Transarterial chemoembolization versus resection for intermediate-stage (BCLC B) hepatocellular carcinoma. Clin Mol Hepatol 2016;22:250-258.

23. Lee EW, Khan S. Recent advances in transarterial embolotherapies in the treatment of hepatocellular carcinoma. Clin Mol Hepatol 2017;23:265-272.

24. Hu HT, Kim JH, Lee LS, et al. Chemoembolization for hepatocellular carcinoma: multivariate analysis of predicting factors for tumor response and survival in a 362-patient cohort. J Vasc Interv Radiol 2011;22:917-923.

25. Miyayama S, Matsui 0. Superselective conventional transarterial chemoembolization for hepatocellular carcinoma: rationale, technique, and outcome. J Vasc Interv Radiol 2016;27:1269-1278.

26. Choi TW, Kim HC, Lee JH, et al. The safety and clinical outcomes of chemoembolization in Child-Pugh class C patients with hepatocellular carcinomas. Korean J Radiol 2015;16:1283-1293.

27. Giannini EG, Moscatelli A, Pellegatta G, et al. Application of the intermediate-stage subclassification to patients with untreated hepatocellular carcinoma. Am J Gastroenterol 2016;111:70-77.

28. Weinmann A, Koch S, Sprinzl M, et al. Survival analysis of proposed BCLC-B subgroups in hepatocellular carcinoma patients. Liver Int 2015;35:591-600.

29. Wang JH, Kee KM, Lin CY, et al. Validation and modification of a proposed substaging system for patients with intermediate hepatocellular carcinoma. J Gastroenterol Hepatol 2015;30:358-363.

30. Yamakado K, Miyayama S, Hirota S, et al. Prognosis of patients with intermediate-stage hepatocellular carcinomas based on the Child-Pugh score: subclassifying the intermediate stage (Barcelona Clinic Liver Cancer stage B). Jpn J Radiol 2014;32:644-649.

31. Kamath PS, Kim WR; Advanced Liver Disease Study Group. The Model for End-Stage Liver Disease (MELD). Hepatology 2007;45:797-805.

32. Malinchoc M, Kamath PS, Gordon FD, Peine CJ, Rank J, ter Borg PC. A model to predict poor survival in patients undergoing transjugular intrahepatic portosystemic shunts. Hepatology 2000;31:864-871.

33. Kamath PS, Wiesner RH, Malinchoc M, et al. A model to predict survival in patients with end-stage liver disease. Hepatology 2001;33:464-470.

34. Dunn W, Jamil LH, Brown LS, et al. MELD accurately predicts mortality in patients with alcoholic hepatitis. Hepatology 2005;41:353-358. 